\title{
Implications of Monte Carlo Statistical Errors in Criticality Safety Assessments
}

\author{
Ronald E. Pevey \\ University of Tennessee, Knoxville
}

15 September 2005

COPYRIGHT NOTICE

This document has been authored by a subcontractor of the U.S. Government under contract DE-AC05-00OR-22800. Accordingly, the U.S. Government retains a paid-up, nonexclusive, irrevocable, worldwide license to publish or reproduce the published form of this contribution, prepare derivative works, distribute copies to the public, and perform publicly and display publicly, or allow others to do so, for U. S. Government purposes.

\section{DISCLAIMER}

This work of authorship and those incorporated herein were prepared by Contractor as accounts of work sponsored by an agency of the United States Government. Neither the United States Government nor any agency thereof, nor Contractor, nor any of their employees, makes any warranty, express or implied, or assumes any legal liability or responsibility for the accuracy, completeness, use made, or usefulness of any information, apparatus, product, or process disclosed, or represents that its use would not infringe privately owned rights. Reference herein to any specific commercial product, process, or service by trade name, trademark, manufacturer, or otherwise, does not necessarily constitute or imply its endorsement, recommendation, or favoring by the United States Government or any agency or Contractor thereof. The views and opinions of authors expressed herein do not necessarily state or reflect those of the United States Government or any agency or Contractor thereof. 


\title{
Implications of Monte Carlo Statistical Errors in Criticality Safety Assessments
}

\author{
Ronald E. Pevey \\ University of Tennessee, Knoxville
}

Prepared for the Safety Analysis Engineering Department

Engineering Division

Y-12 National Security Complex

Oak Ridge, Tennessee, 37831-8238

by the University of Tennessee, Knoxville under subcontract 4300020173

Managed by BWXT L.L.C.

for the U.S. Department of Energy

under contract DE-AC05-00OR22800. 
Y/DD-1191

\title{
Implications of Monte Carlo Statistical Errors in Criticality Safety Assessments
}

\begin{abstract}
Most criticality safety calculations are performed using Monte Carlo techniques because of Monte Carlo's ability to handle complex three-dimensional geometries. For Monte Carlo calculations, the more histories sampled, the lower the standard deviation of the resulting estimates. The common intuition is, therefore, that the more histories, the better; as a result, analysts tend to run Monte Carlo analyses as long as possible (or at least to a minimum acceptable uncertainty). For Monte Carlo criticality safety analyses, however, the optimization situation is complicated by the fact that procedures usually require that an extra margin of safety be added because of the statistical uncertainty of the Monte Carlo calculations. This additional safety margin affects the impact of the choice of the calculational standard deviation, both on production and on safety. This paper shows that, under the assumptions of normally distributed benchmarking calculational errors and exact compliance with the upper subcritical limit (USL), the standard deviation that optimizes production is zero, but there is a non-zero value of the calculational standard deviation that minimizes the risk of inadvertently labeling a supercritical configuration as subcritical. Furthermore, this value is shown to be a simple function of the typical benchmarking step outcomes - the bias, the standard deviation of the bias, the upper subcritical limit, and the number of standard deviations added to calculated keffectives before comparison to the USL.
\end{abstract}

\section{INTRODUCTION}

The traditional procedure of determining criticality safety limits for a proposed operation involving fissile nuclear material is governed by ANSI/ANS-8.1-1198 [Ref. 1]. The traditional interpretation of the requirements of this standard (e.g., Ref. 2) leads to a twostep procedure to establish the maximum mass of fissile material that can be loaded into a proposed configuration: (1) determine a maximum allowed calculated value of k-effective ("upper subcritical limit" or USL), specific to the methodology used; and (2) translate this 
criticality limit into an upper limit on the mass of fissile material that the configuration may contain. Before defining the problem to be addressed, a brief description will be given of this two-step procedure.

The first step is done to validate the use of the calculational methodology for analyzing the proposed configuration. This is accomplished by calculating k-effective $\left(k_{e f f}\right)$ for a number of known critical experiments (i.e., with the true $k_{\text {eff }}$ known to be 1.00) that are similar to the proposed configuration and then performing a statistical analysis on the resulting computational errors. The statistical analysis results in an estimate of the methodology's bias for this type of configuration and an estimate of the standard deviation of the bias. These benchmark errors will have contributions not only from calculational approximations, but also from experiment dimension uncertainties, material content uncertainties, neutron cross section data shortcomings, etc. [Ref. 3]. Using the bias, the standard deviation of the bias, and a good deal of engineering judgment, the analyst sets an upper subcritical limit ("USL")—a value of $k_{\text {eff }}$ for which any configuration with a higher computed $k_{\text {eff }}$ would be assumed to be supercritical, and thus not allowed.

For example, if a series of appropriately chosen critical experiments is calculated by the methodology and found to have computed $k_{\text {eff }}$ values ranging from 0.98 to 1.02 , the analyst might decide that any configuration whose computed $k_{\text {eff }}$ is below 0.97 might reasonably be assumed to be subcritical. (If the statistical analysis for the benchmark calculations results in an average $k_{\text {eff }}$ value of 0.995 with a standard deviation of 0.005 , the USL of 0.97 would represent a limit that is five experimental standard deviations below the average value.)

The subsequent analysis step involves the translation of the USL into a maximum allowable mass of fissile material by performing a search for the fissile mass loading for 
which the calculated $k_{\text {eff }}$ is equal to the USL. This step employs the same calculational methodology that was used in the benchmarking step to estimate the $k_{\text {eff }}$ value of the most reactive credible operational configuration (usually identified through a detailed process upset contingency analysis).

Most criticality safety calculations are performed using Monte Carlo techniques because of their ability to handle complex three-dimensional geometries. When the analysis methodology is a Monte Carlo calculation, the analyst must-in addition to modeling the geometry and materials of the configuration—also choose a desired calculational standard deviation to use for the analysis, which is then implemented by selecting the appropriate number of neutron histories to be used in the analysis. Since such a Monte Carlo calculation results in a statistical uncertainty in the resulting $k_{\text {eff }}$ prediction, it is the usual practice to add two calculational standard deviations to the calculated $k_{\text {eff }}$ before comparing to the USL ("sigma penalty") to compensate for this added uncertainty. (The effect is identical to lowering the USL by two calculational standard deviations.)

For instance, for the previous example of an established USL of 0.97 the analyst could choose a calculational standard deviation of 0.01 and not allow a Monte Carlo-predicted $k_{\text {eff }}$ of more than 0.95 ; or the calculational standard deviation could be cut in half to 0.005 with a calculated $k_{\text {eff }}$ of 0.96 or lower deemed acceptable. The smaller the calculational standard deviation, obviously, the closer the calculated $k_{\text {eff }}$ can be to the USL. Since the standards do not specify the calculated standard deviation to use in criticality safety analyses, an individual analyst is free to choose this value.

The purpose of this paper is to examine the situation faced by an analyst trying to optimize the choice of the calculational standard deviation. An important assumption (which 
mirrors the real-life situation) is that this is a constrained optimization problem. We will assume that the USL has been determined through a process that balances production and safety, and that the analyst is constrained to exactly comply with the limit, i.e., that the limit can be neither increased to allow more production nor decreased to enhance safety. The analyst is at the point of choosing a calculational standard deviation and then performing a search for the maximum fissile mass limit consistent with the pre-determined USL.

\section{OPTIMIZATION OPTION \#1: MAXIMIZE FISSILE MASS LIMIT}

Since the USL is set through a balance of production and safety, we will first examine the situation where the analyst chooses the calculational standard deviation to maximize production, i.e., to produce the highest possible fissile mass limit.

Because the procedures usually require that the sigma penalty be applied for Monte Carlo calculation techniques, the analyst's job is to find the fissile mass that results in a calculated $k_{\text {eff }}$ given by:

$$
\lambda_{\text {calc }}=U S L-2 \sigma_{\text {calc }}
$$

This optimization path has an easily stated (but unattainable) optimal calculational standard deviation of zero, based on the fact that a lower $\sigma_{\text {calc }}$ results in a larger allowable $k_{\text {eff }}$ value, which, on average, results in a larger allowable fissile mass.

Of course, it is well known that the smaller the target standard deviation, the longer that the Monte Carlo calculations will have to run, adding to the expense of the analysis (with a factor of $x$ reduction of the standard deviation typically requiring a factor of $x^{2}$ increase in computer time). So, the optimization option of maximum production leads to the common situation where additional production can be obtained only at additional cost. This situation requires a cost/benefit analysis that depends on the specifics of the analysis: number of 
calculations required, amount and cost of resources, etc. In the end, a specific determination must be made of the value of the incremental fissile mass versus the cost of the incremental resources required to justify it.

\section{OPTIMIZATION OPTION \#2: MAXIMIZE SAFETY}

A second optimization option is to choose the calculational standard deviation that maximizes safety (under the padding procedure previously described in Section I). Since the purpose of criticality analysis is to assure that processes involving fissile material remain subcritical, we will define our safety goal to be the minimization of the probability of inadvertently hbeling a supercritical configuration as subcritical (“criticality risk"). Note that no claim is made that this maximizes safety under all possible procedures, just under the padding procedure in Section 1. (Indeed, this paper will suggest an even safer, though perhaps not desirable in practice, procedure after the optimum sigma for the procedure in Section I is derived.)

In analyzing this probability, we should consider that the crit icality risk has two parts:

(1) The danger that the physical configuration is one for which the computational tool will systematically underestimate the true $k_{\text {eff; }}$ and

(2) The danger that a single stochastic calculation of $k_{\text {eff }}$ will underestimate the expected value of $k_{\text {eff }}$ of the configuration from the computational tool.

With this in mind, a reduction of the calculational standard deviation will have two conflicting effects on the criticality risk: the increased precision of the calculation reduces the danger of (2) above (by limiting the variance of the calculational results) while at the same time increasing the danger of (1) by allowing a more reactive configuration to be accepted (because of the higher value of USL- $2 \sigma_{\text {calc }}$ due to a reduction of the sigma penalty). Since 
we have these competing effects, the possibility exists that there is a non-zero choice of the calculational standard deviation that optimally balances these two dangers, while exactly complying with the USL.

The validation procedure uses calculational estimates of a known set of criticality benchmarks. The calculational method's ability to estimate $k_{\text {eff }}$ for the set can be quantified using:

$$
\begin{gathered}
\lambda_{\text {exp }, i} \equiv \text { Known } k_{\text {eff }} \text { value for experiment } i=1.00 \\
\lambda_{\text {calc }, i} \equiv \text { Calculatio nal estimate of } k_{\text {eff }} \text { value for } \\
\text { experiment } i \\
b_{i} \equiv \text { Error of calculatio n of experiment } i=\lambda_{\text {calc }, i}-\lambda_{\text {exp }, i} \\
\hat{b} \equiv \text { Estimate of expected bias of the calculatio nal method } \\
=E\left[\lambda_{\text {calc }, i}-\lambda_{\text {exp }, i}\right] \equiv E\left[b_{i}\right] \\
S_{b} \equiv \text { Estimate of standard deviation of the } \\
\text { bias } \equiv \sqrt{E\left[b_{i}^{2}\right]-E^{2}\left[b_{i}\right]}
\end{gathered}
$$

Assuming that $\mathrm{N}$ experiments are used in the validation, statistical estimates of bias and its standard deviation can be determined using:

$$
\hat{b}=\frac{\sum_{i=1}^{N} \lambda_{c a l c, i}-\lambda_{\text {exp }, i}}{N}=\frac{\sum_{i=1}^{N} b_{i}}{N}
$$

and

$$
S_{b}=\sqrt{\frac{\sum_{i=1}^{N}\left(b_{i}-\hat{b}\right)^{2}}{N-1}}
$$


If, as is often the case, the bias distribution passes a test for normality, the underlying distribution of bias can be estimated using the normal distribution:

$$
p(b)=\frac{1}{\sqrt{2 \pi} S_{b}} e^{-(b-\hat{b})^{2} / 2 S_{b}^{2}}
$$

We interpret this equation to mean that if the same calculational method were used to estimate the $k_{\text {eff }}$ of a similar physical system, and the result of the calculation were $\lambda_{\text {calc }}$, that the expected distribution of the true $k_{\text {eff }}, \lambda_{\text {exp }}$, of the configuration would depend on $b$ according to:

$$
\operatorname{Pr}\left\{\lambda_{\text {exp }}(b) \in\left(\lambda_{\text {calc }}-b, \lambda_{\text {calc }}-b+d b\right)\right\}=p(b) d b
$$

where $b$ is distributed according to $p(b)$ in Equation 1.

Using this distribution for the bias, the probability that the system is supercritical is given by:

$$
\begin{aligned}
\operatorname{Pr}\{\text { criticalit } \mathrm{y}\} & =\operatorname{Pr}\left\{\lambda_{\text {exp }}(b)>1\right\} \\
= & \operatorname{Pr}\left\{\lambda_{\text {calc }}-b>1\right\} \\
& =\operatorname{Pr}\left\{b<\lambda_{\text {calc }}-1\right\} \\
& =\int_{-\infty}^{\lambda_{\text {cal }}-1} p(b) d b \\
& =\int_{-\infty}^{\lambda_{\text {cal }}-1} \frac{1}{\sqrt{2 \pi} S_{b}} e^{-(b-\hat{b})^{2} / 2 S_{b}^{2}} d b
\end{aligned}
$$

We can reduce the probability of criticality by decreasing $\lambda_{\text {calc }}-1$, which is generally accomplished through the establishment of an upper subcritical limit (USL) above which $\lambda_{\text {calc }}$ is not permitted.

If the above distribution is rewritten in terms of the variable $z$, where:

$$
z \equiv \frac{\hat{b}-b}{S_{b}} ; d z=-\frac{d b}{S_{b}},
$$


the resulting probability is:

$$
\begin{gathered}
\operatorname{Pr}\left\{\text { criticalit } \mathrm{y} \mid \lambda_{\text {calc }}\right\}=\int_{\frac{(1+\hat{b})-\lambda_{\text {calc }}}{S_{b}}}^{\infty} \frac{1}{\sqrt{2 \pi}} e^{-z^{2} / 2} d z \\
<\int_{\frac{(1+\hat{b})-U S L}{S_{b}}}^{\infty} \frac{1}{\sqrt{2 \pi}} e^{-z^{2} / 2} d z
\end{gathered}
$$

The last lower limit in the above integral is a measure of how far the USL is below the expected calculated $k_{\text {eff }}$ for critical systems, expressed in units of the standard deviation of the bias, $S_{b}$. We will refer to this number as $n$ :

$$
\begin{aligned}
n \equiv & \text { Number of bias uncertaint y units that the USL is } \\
& \text { conservati vely defined below the expected } \\
& \text { calculated } k_{\text {eff }} \text { for critical systems } \\
= & \frac{(1+\hat{b})-U S L}{S_{b}}
\end{aligned}
$$

(Note that we are not stating that the USL is actually established by specifying a value of $n$; we are only saying that once a USL has been established, the value of $n$ can be found from the above equation.) From standard tables of normal probability distributions ${ }^{4}$, we can approximate the probability of criticality as a function of $n$ using Table I.

The development thus far is for a deterministic method of calculating $k_{\text {eff. }}$ If the method for calculating $k_{\text {eff }}$ is a Monte Carlo method, the analyst must decide on the number of Monte Carlo histories that are used in the Monte Carlo calculation. This choice affects the precision of the resulting $k_{\text {eff }}$ estimate, which is presented by the code as an expected value of $k_{\text {eff }}$ and a normally distributed standard deviation, i.e.,

$$
k_{\text {eff }} \cong \hat{\lambda}_{\text {calc }} \pm \sigma_{\text {calc }}
$$


(Note that we assume that the number of standard deviations is chosen to produce a predetermined target calculational standard deviation, $\sigma_{\text {calc }}$. Because of this, the $\sigma_{\text {calc }}$ is not treated as a stochastic variable and therefore not labeled with a caret.)

We interpret the previous equation to mean that repeated uses of the same method (with statistical independence) would result in an expected value of the calculated $k_{\text {eff }}$ that follows a normal distribution centered on the one calculation that was actually performed:

$$
p\left(\lambda_{\text {calc }}\right)=\frac{1}{\sqrt{2 \pi} \sigma_{\text {calc }}} e^{-\left(\lambda_{\text {calc }}+\hat{\lambda}_{\text {calc }}\right)^{2} / 2 \sigma_{\text {calc }}^{2}}
$$

Using this distribution of $\lambda_{\text {calc }}$ values (rather than the fixed value assumed before) changes the probability of criticality in the following way:

$$
\begin{aligned}
& \operatorname{Pr}\{\text { criticalit } \mathrm{y}\}=\int_{-\infty}^{\infty} \operatorname{Pr}\left\{\text { criticalit } \mathrm{y} \mid \lambda_{\text {calc }}\right\} p\left(\lambda_{\text {calc }}\right) d \lambda_{\text {calc }} \\
&=\int_{-\infty}^{\infty} \frac{1}{\sqrt{2 \pi} \sigma_{\text {calc }}} e^{-\left(\lambda_{\text {calc }}-\hat{-}_{\text {calc }}\right)^{2} / 2 \sigma_{\text {calc }}^{2}} \\
& \times \int_{\frac{(1+\hat{b})-\lambda_{\text {calc }}}{S_{b}}}^{\infty} \frac{1}{\sqrt{2 \pi}} e^{-z^{2} / 2} d z d \lambda_{\text {calc }}
\end{aligned}
$$

If we change the first variable of integration to:

$$
y=\frac{\lambda_{\text {calc }}-\hat{\lambda}_{\text {calc }}}{\sigma_{\text {calc }}} ; d y=\frac{d \lambda_{\text {calc }}}{\sigma_{\text {calc }}}
$$

the result becomes:

$$
\begin{aligned}
\operatorname{Pr}\{\text { criticalit } \mathrm{y}\}= & \int_{-\infty}^{\infty} \frac{1}{\sqrt{2 \pi}} e^{-y^{2} / 2} \\
& \times \int_{\frac{(1+\hat{b})-\hat{x}_{\text {calc }}-y \sigma_{\text {calc }}}{S_{b}}}^{\infty} \frac{1}{\sqrt{2 \pi}} e^{-z^{2} / 2} d z d y
\end{aligned}
$$


The integrals are standard; the complexity is buried in the lower limit of the second integral. The earlier definition of $n$ relates the variables $(1+\hat{b}), S_{b}$, and the USL. Standard criticality practice relates the other two variables to the USL in the following way.

When Monte Carlo calculations are used to predict the $k_{\text {eff }}$ value of proposed operations, it is standard practice to allow for the statistical variability of the Monte Carlo results by requiring that

$$
\hat{\lambda}_{\text {calc }}+2 \sigma_{\text {calc }} \leq U S L
$$

i.e., to add two standard deviations to the Monte Carlo estimate of $k_{\text {eff }}$ before comparing to the USL. We refer to this as the "sigma penalty."

This common practice can be generalized by defining a variable $m$ :

$$
\begin{aligned}
m \equiv \text { Number of calculational standard deviations added to } \hat{\lambda}_{\text {calc }} \text { before } \\
\\
\text { comparing to the USL }
\end{aligned}
$$

The above equation and the earlier definition of the variable $n$ combine to give two equations for the USL:

$$
\hat{\lambda}_{\text {calc }}+m \sigma_{\text {calc }} \leq U S L=(1+\hat{b})-n S_{b}
$$

Our previous constraint on the analyst to find the maximum fissile material content that would pass the test is equivalent to assuming equality in the left-hand equation. With this assumption, the lower limit of Eqn. 2 can be written in terms of $m$ and $n$ :

$$
\begin{aligned}
\operatorname{Pr}\{\text { criticalit } \mathrm{y}\}= & \int_{-\infty}^{\infty} \frac{1}{\sqrt{2 \pi}} e^{-y^{2} / 2} \\
& \times \int_{n+(m-y) \frac{\sigma_{\text {calc }}}{S_{b}}}^{\infty} \frac{1}{\sqrt{2 \pi}} e^{-z^{2} / 2} d z d y
\end{aligned}
$$


This result indicates that, for values of $n$ and $m$ defined for a given criticality safety analysis, the risk of criticality due to bad luck (i.e., statistical variation in the spread of benchmark errors and statis tical variation of the analysis results) depends on the ratio of the chosen calculational standard deviation used in the analysis and the fixed, pre-determined standard deviation of the bias.

Defining this ratio as $r$, i.e.,

$$
r=\frac{\sigma_{c a l c}}{S_{b}}
$$

gives (for fixed $m$ and $n$ ):

$$
\begin{aligned}
& f_{m n}(r) \equiv \operatorname{Pr}\{\text { criticalit } \mathrm{y}\} \\
&=\int_{-\infty}^{\infty} \frac{1}{\sqrt{2 \pi}} e^{-y^{2} / 2} \int_{n+(m-y) r}^{\infty} \frac{1}{\sqrt{2 \pi}} e^{-z^{2} / 2} d z d y
\end{aligned}
$$

Note that for $r=0$, this reduces to the deterministic probability given by Eqn. 2 .

As was mentioned previously, a typical value for $m$ is 2 ; a typical value for $n$ in an actual criticality safety evaluation is about 5-10 (e.g., critically benchmarks calculated near $k_{\text {eff }}=1.00$ with $S_{b} \cong 0.5-1 \%$ and $U S L \cong 0.95$ ).

Figure 1 (developed from a numerical evaluation of Equation 4) shows the dependence of criticality probability on $r$ for various values of $m$ given $n=5$. As can be seen, the probability does not depend on $m$ for $r=0$, which corresponds to an ideal, infinite-history, zero-variance Monte Carlo calculation. The $m=0$ case, which corresponds to the case where there is no sigma penalty, results in monotonically increasing criticality danger as $\sigma_{\text {calc }}$ increases_-justifying the need for a sigma penalty to compensate for this extra risk.

For all cases with positive $m$ (i.e., a sigma penalty), the criticality risk drops with increasing $\sigma_{\text {calc }}$ to a minimum, then increases. This is an important result because it 
indicates that the sigma penalty is performing its intended purpose-compensating for the extra criticality risk due to calculational uncertainty in $k_{\text {eff }}$ - as long as $\sigma_{\text {calc }}$ is less than the minimum of the curve, but not beyond the minimum. This suggests that there may be some benefit in finding the value of $\sigma_{\text {calc }}$ that minimizes the criticality risk.

The minimum of Equation 4 can be found in the usual way-by finding the value of $r$ that makes the derivative equal to zero:

$$
\begin{gathered}
\frac{d f_{m n}(r)}{d r}=\frac{d}{d r}\left[\int_{-\infty}^{\infty} \frac{1}{\sqrt{2 \pi}} e^{-y^{2} / 2} \int_{n+(m-y) r}^{\infty} \frac{1}{\sqrt{2 \pi}} e^{-z^{2} / 2} d z d y\right] \\
=\int_{-\infty}^{\infty} \frac{1}{\sqrt{2 \pi}} e^{-y^{2} / 2} \frac{d}{d r}\left[\int_{n+(m-y) r}^{\infty} \frac{1}{\sqrt{2 \pi}} e^{-z^{2} / 2} d z\right] d y \\
=\int_{-\infty}^{\infty} \frac{1}{\sqrt{2 \pi}} e^{-y^{2} / 2}\left[-\frac{1}{\sqrt{2 \pi}} e^{-(n+(m-y) r)^{2} / 2} \frac{d}{d r}(n+(m-y) r)\right] d y \\
=\int_{-\infty}^{\infty} \frac{1}{\sqrt{2 \pi}} e^{-y^{2} / 2}\left[-\frac{1}{\sqrt{2 \pi}} e^{-(n+(m-y) r)^{2} / 2}(m-y)\right] d y \\
=-\frac{1}{2 \pi} \int_{-\infty}^{\infty}(m-y) e^{-\left[\left(1+r^{2}\right) y^{2}-2(n+m r) r y+(n+m r)^{2}\right] / 2} d y=0
\end{gathered}
$$

Since the exponential argument inside the last integral is a parabola with equation:

$$
z(y)=-\frac{\left(1+r^{2}\right) y^{2}-2(n+m r) r y+(n+m r)^{2}}{2},
$$

there is an axis of symmetry through the vertex of the parabola, which is found by setting the derivative of the parabola to zero and solving for $y$ to get:

$$
y=y_{a}=\frac{(n+m r) r}{1+r^{2}}
$$


Changing the variable of integration in Equation 5 from y to $w=y-y_{a}$ and dividing the integral into two parts yields:

$$
\begin{gathered}
\frac{d f_{m n}(r)}{d r}=\frac{-1}{2 \pi} e^{-\left[\frac{(n+m r)^{2}}{2\left(1+r^{2}\right)}\right]} \int_{-\infty}^{\infty}\left(\left(m-y_{a}\right)-w\right) e^{-\left(1+r^{2}\right) w^{2} / 2} d w \\
=-\frac{m-y_{a}}{2 \pi} e^{-\left[\frac{(n+m r)^{2}}{2\left(1+r^{2}\right)}\right]} \int_{-\infty}^{\infty} e^{-\left(1+r^{2}\right) w^{2} / 2} d w \\
+\frac{1}{2 \pi} e^{-\left[\frac{(n+m r)^{2}}{2\left(1+r^{2}\right)}\right]} \int_{-\infty}^{\infty} w e^{-\left(1+r^{2}\right) w^{2} / 2} d w=0
\end{gathered}
$$

The second term is equal to zero because it involves a symmetric-domain integral of the product of an odd function and an even function. In addition, use of:

$$
\int_{-\infty}^{\infty} e^{-x^{2} / 2 \sigma^{2}} d x=\sqrt{2 \pi} \sigma
$$

allows simplification of the first integral to give us:

$$
\frac{d f_{m n}(r)}{d r}=-\left(m-y_{a}\right)\left[\frac{e^{-\frac{(n+m r)^{2}}{2\left(1+r^{2}\right)}}}{\sqrt{2 \pi\left(1+r^{2}\right)}}\right]=0
$$

Since the term in brackets is positive for non-zero values of $m, n$, and $r$, it can be factored out to reveal an extremum at the value of $r$ for which:

$$
m=y_{a}=\frac{(n+m r) r}{1+r^{2}}
$$

This can be solved for $r$ to yield an extremum at:

$$
r=\frac{m}{n}
$$


This extremum can be shown to be a minimum by evaluating the second derivative of $f_{m n}(r)$ at the extremum. If we substitute $y_{a}$ from Equation 6 into Equation 7, we can write the first derivative using the product of two functions:

$$
\frac{d f_{m n}(r)}{d r}=-a(r) b(r)
$$

where:

$$
\begin{gathered}
a(r)=\left(m-y_{a}\right)=\left(m-\frac{(n+m r) r}{1+r^{2}}\right)=\frac{m-n r}{1+r^{2}} \\
b(r)=\frac{e^{\frac{(n+m r)^{2}}{2\left(1+r^{2}\right)}}}{\sqrt{2 \pi\left(1+r^{2}\right)}}
\end{gathered}
$$

If we differentiate this again and evaluate at the extremum, we get:

$$
\left.\frac{d^{2} f_{m n}(r)}{d r^{2}}\right|_{r=\frac{m}{n}}=-a\left(\frac{m}{n}\right)^{d b\left(\frac{m}{n}\right)} \frac{d\left(\frac{m}{n}\right)}{d r} \frac{d a\left(\frac{m}{n}\right)}{d r}
$$

Since

$$
a\left(\frac{m}{n}\right)=\frac{m-n \frac{m}{n}}{1+\left(\frac{m}{n}\right)^{2}}=\frac{m-m}{1+\left(\frac{m}{n}\right)^{2}}=0
$$

and

$$
\frac{d a(r)}{d r}=\frac{n r^{2}-2 r m-n}{\left(1+r^{2}\right)^{2}}
$$

therefore 


$$
\frac{d a\left(\frac{m}{n}\right)}{d r}=\frac{n\left(\frac{m}{n}\right)^{2}-2\left(\frac{m}{n}\right) m-n}{\left(1+\left(\frac{m}{n}\right)^{2}\right)^{2}}=\frac{\frac{n m^{2}-2 n m^{2}-n^{3}}{n^{2}}}{\frac{\left(m^{2}+n^{2}\right)^{2}}{n^{4}}}=\frac{-n^{3}\left(m^{2}+n^{2}\right)}{\left(m^{2}+n^{2}\right)^{2}}=\frac{-n^{3}}{m^{2}+n^{2}}
$$

and

$$
b\left(\frac{m}{n}\right)=\frac{e^{-\left[\frac{\left(n+m\left(\frac{m}{n}\right)\right)^{2}}{2\left(1+\left(\frac{m}{n}\right)^{2}\right)}\right]}}{\sqrt{2 \pi\left(1+\left(\frac{m}{n}\right)^{2}\right)}}=\frac{n e^{-\left(m^{2}+n^{2}\right) / 2}}{\sqrt{2 \pi\left(m^{2}+n^{2}\right)}} .
$$

The second derivative at the extremum is therefore given by:

$$
\left.\frac{d^{2} f_{m n}(r)}{d r^{2}}\right|_{r=\frac{m}{n}}=\frac{n^{4} e^{-\left(m^{2}+n^{2}\right) / 2}}{\sqrt{2 \pi\left(m^{2}+n^{2}\right)^{3}}}
$$

This is positive for all nonzero $m$ and $n$. Therefore, the extremum is a minimum for all nonzero $m$ and $n$.

The result of all of this is found by combining Equation 3 and Equation 8, to reveal that the probability of inadvertent criticality is minimized if the analyst performs the Monte Carlo calculation using a calculational standard deviation given by:

$$
\sigma_{\text {calc }}=\left(\frac{m}{n}\right) S_{b}
$$

Referring again to Figure 1, we can see that the curves plotted appear consistent with this result, with minima apparently near values of $m / 5$ for each curve.

These results indicate that:

(1) Calculational standard deviations above the value in Equation 9 should be used with care, since beyond this value the marginal increase in the safety margin from the sigma 
penalty is not adequate to compensate for the marginal increase in criticality risk introduced by the less precise $k_{\text {eff }}$ calculation.

(2) In contrast, for calculational standard deviations from zero to the value in Equation 9, the criticality risk decreases with increasing $\sigma_{\text {calc }}$ due to the fact that the marginal increase in the safety margin provided by the sigma penalty more than compensates for the marginal increase in criticality risk caused by the extra uncertainty in the $k_{\text {eff }}$ calculation.

Note that this paper is not claiming that a higher error Monte Carlo calculation is preferable to a lower error Monte Carlo calculation, just that analysts should rest assured that the sigma penalty is providing its intended effect as long as $\sigma_{\text {calc }}$ is less than the value indicated in Equation 9. In fact, a better safety assessment would result from utilizing a $\sigma_{\text {calc }}$ less than the value in Equation 9 and adding $m$ times the Equation 9 value (rather than the actual $\sigma_{\text {calc }}$ used in the calculation) to the calculated $k_{\text {eff }}$ before comparison to the USL. This has the added advantage of using a better Monte Carlo estimate of $k_{\text {eff }}$ while retaining the reduced criticality probability offered by the optimum $\sigma_{\text {calc }}$.

\section{SAMPLE PROBLEM}

To illustrate the use of these results, the "34 Fresh Fuel LWR-type Criticals" sample problem was borrowed from Ref. 5. In this problem the 29 values of $k_{\text {eff }}$ shown in Table II are extracted from relevant critical benchmarks and statistically analyzed.

Assuming (as stated in the reference) that these data have passed a normality test, the resulting ave rage and standard deviation are given by:

$$
\begin{aligned}
& \hat{b}=0.99975-1.0=-0.00025 \\
& S_{b}=0.009392
\end{aligned}
$$


It is common practice to define the "bias uncertainty" mentioned in Ref. 1 as 2.5 to 3 of these experimental standard deviations. Use of the high end of this range $(n=3)$ would result in an upper subcritical limit of 0.9715 . If, as is common practice, the value of $m$ is set at 2, the calculational standard deviation that minimizes the criticality risk would be given by:

$$
\sigma_{\text {calc }}=\left(\frac{m}{n}\right) S_{b}=0.00626
$$

This is a somewhat higher value of the calculational standard deviation than is usual, indicating that typical $\sigma_{\text {calc }}$ values are within the range where the sigma penalty is effectively reducing risk. Note that if the USL is reduced to $0.95-$ which is a common reduction due to site-specific "minimum subcritical margins"- then the optimal value drops to:

$$
\begin{aligned}
& n=\frac{(1+\hat{b})-0.95}{S_{b}}=\frac{0.99975-0.95}{0.009392}=5.30 \\
& \sigma_{\text {calc }}=\left(\frac{m}{n}\right) S_{b}=\left(\frac{2}{5.3}\right) 0.009392=0.0035
\end{aligned}
$$

which is more in line with normal usage (and even corresponds closely to the $\sigma_{\text {calc }}$ used in Ref. 5).

\section{CONCLUSIONS}

The purpose of this development is to examine the effect of the choice of calculational standard deviation in Monte Carlo criticality safety calculations aimed at determining fissile mass limits.

The first option, choosing the standard deviation to maximize these fissile mass limits, was found to lead to the typical Monte Carlo trade-off: the lower the calculational standard deviation, the better. This was shown to involve situation-specific marginal cost/benefit analysis for which nothing more could generally be said. 
The second option, choosing the calculational standard deviation to minimize the probability of misjudging a physically critical configuration to be subcritical, was shown to lead to a non-zero optimal calculational standard deviation. The development shows thatunder the assumption of normally distributed calculational biases of the criticality benchmarks and exact compliance with the USL- for any $m>0$ the criticality danger decreases as $\sigma_{\text {calc }}$ increases to a minimum at:

$$
\sigma_{\text {calc }}=\left(\frac{m}{n}\right) S_{b}
$$

which is based on the USL and known benchmarking statistical results.

In a real analysis situation, both results might be useful. Even if the analyst's goal is to maximize the fissile material mass limit, the economic reality is that a non-zero calculational standard deviation must ultimately be used in the Monte Carlo calculations. If the chosen $\sigma_{\text {calc }}$ less than $\left(\frac{m}{n}\right) S_{b}$, the criticality analyst can be assured that the sigma penalty adequately compensates for the danger introduced by using a non-zero $\sigma_{\text {calc }}$ in the Monte Carlo calculations.

\section{ACKNOWLEDGEMENTS}

The author would like to thank Dr. Peter Groer of the University of Tennessee Nuclear Engineering Department for his review of the text. In addition, the author would like to express his appreciation to an anonymous reviewer who contributed greatly to the clarity of the paper. 


\section{REFERENCES}

1. "American National Standard for Nuclear Criticality Safety in Operations with Fissionable Materials Outside Reactors," ANSI/ANS-8.1-1998, American Nuclear Society Standards Committee Working Group ANS-8.1 (Sep. 1998).

2. R. H. SMITH, H. J. KEENER, J. F. DECLUE, A. W. KRASS, "Methodology for Determination of the Upper Subcritical Limit for Criticality Calculations for Criticality Safety Analyses,” Y/DD-972, Y-12 National Security Co mplex (Apr. 2001).

3. B. L. BROADHEAD, C. M. HOPPER, R. L. CHILDS, C. V. PARKS, "Sensitivity and Uncertainty Analyses Applied to Criticality Safety Validation," NUREG/CR-6655, Vol. 1, Oak Ridge National Laboratory (Oct. 1999).

4. M. ABROMOWITZ, I. A. STEGUN (ed.), "Handbook of Mathematical Functions With Formulas, Graphs, and Mathematical Tables,” National Bureau of Standards Applied Mathematical Series 55 (1966).

5. M. D. DEHART, "User's Manual for USLSTATS V1.0." From "Criticality Benchmark Guide for LightWater-Reactor Fuel in Transportation and Storage Packages," NUREG/CR-6361, Appendix C, Oak Ridge National Laboratory (Jul. 2000). 
TABLE I

. Probability of criticality vs. $\mathrm{n}$ for deterministic criticality calculational methods

\begin{tabular}{|c|c|}
\hline $\mathbf{n}$ & $\operatorname{Pr}\{$ criticality $\mid \mathbf{n}\}=\mathbf{Q}(\mathbf{n})^{a}$ \\
\hline 0 & $5.0000-1^{b}$ \\
\hline 1 & $1.5866-1$ \\
\hline 2 & $2.2750-2$ \\
\hline 3 & $1.3499-3$ \\
\hline 4 & $3.1671-5$ \\
\hline 5 & $2.8665-7$ \\
\hline 6 & $9.8660-10$ \\
\hline 7 & $1.2798-12$ \\
\hline
\end{tabular}


Table II

Sample problem benchmark k-effective values(from Ref. 5)

\begin{tabular}{|c|c|c|c|}
\hline $\begin{array}{c}\text { Bench } \\
\text { mark }\end{array}$ & $\boldsymbol{k}_{\text {eff }}$ & $\begin{array}{c}\text { Bench } \\
\text { mark }\end{array}$ & $\boldsymbol{k}_{\text {eff }}$ \\
\hline 1 & 0.99647 & 16 & 1.00874 \\
\hline 2 & 0.99776 & 17 & 1.0119 \\
\hline 3 & 1.00764 & 18 & 1.0098 \\
\hline 4 & 0.99587 & 19 & 1.00565 \\
\hline 5 & 0.99744 & 20 & 1.01929 \\
\hline 6 & 1.00337 & 21 & 1.0086 \\
\hline 7 & 0.99609 & 22 & 0.99487 \\
\hline 8 & 1.00108 & 23 & 0.99257 \\
\hline 9 & 0.99737 & 24 & 1.00132 \\
\hline 10 & 0.98408 & 25 & 0.99154 \\
\hline 11 & 0.98871 & 26 & 1.00028 \\
\hline 12 & 0.99527 & 27 & 0.99565 \\
\hline 13 & 0.98804 & 28 & 0.98574 \\
\hline 14 & 1.01363 & 29 & 0.98733 \\
\hline 15 & 1.0166 & & \\
\hline
\end{tabular}




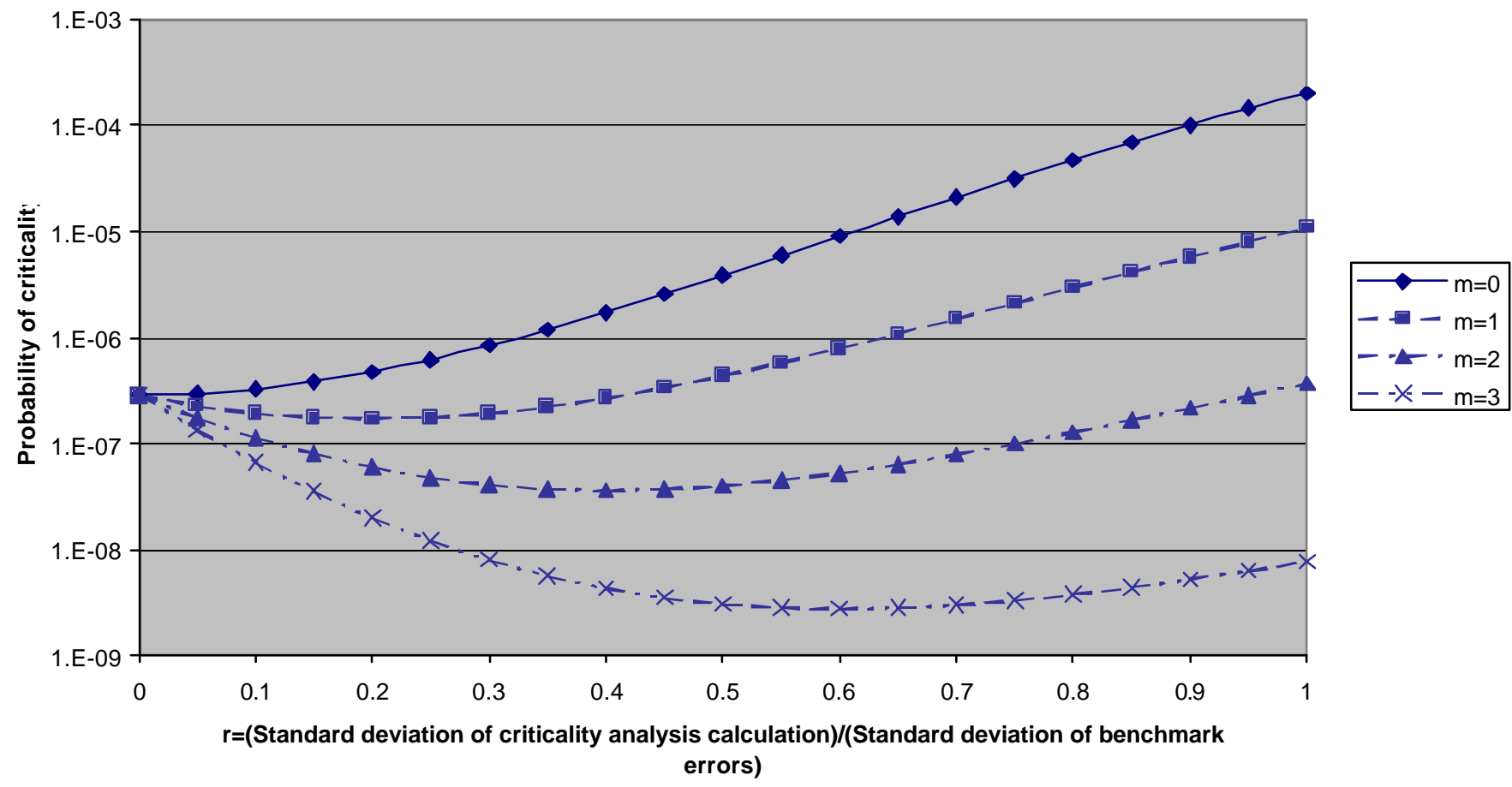

Fig. 1. Probability of Criticality as a Function of Calculational Standard Deviation (for $n=5) F$ 